Revue des patrimoines

$31 \mid 2017$

Patrimoines de la santé : essais de définition - enjeux de conservation

\title{
Patrimoines de la santé : essais de définition - enjeux de conservation \\ Éditorial
}

Isabelle Duhau et Cécile Lestienne

\section{(2) OpenEdition \\ Journals}

\section{Édition électronique}

URL : http://journals.openedition.org/insitu/14481

DOI : 10.4000/insitu. 14481

ISSN : $1630-7305$

\section{Éditeur}

Ministère de la Culture

\section{Référence électronique}

Isabelle Duhau et Cécile Lestienne, «Patrimoines de la santé : essais de définition - enjeux de

conservation », In Situ [En ligne], 31 | 2017, mis en ligne le 01 mars 2017, consulté le 10 octobre 2020

URL : http://journals.openedition.org/insitu/14481 ; DOI : https://doi.org/10.4000/insitu.14481

Ce document a été généré automatiquement le 10 octobre 2020.

\section{cc) $(1) \odot$}

In Situ Revues des patrimoines est mis à disposition selon les termes de la licence Creative Commons Attribution - Pas d'Utilisation Commerciale - Pas de Modification 4.0 International. 


\title{
Patrimoines de la santé : essais de définition - enjeux de conservation
} Éditorial

\author{
Isabelle Duhau et Cécile Lestienne
}

1 Ce numéro d'In Situ. Revue des patrimoines consacré aux patrimoines de la santé - le premier traitant de ce sujet - trouve son origine dans l'actualité. Les recherches sur l'architecture hospitalière se sont multipliées ces dernières années. Elles ont donné lieu à deux importantes synthèses, celle, régionale, publiée par le service Patrimoine et Inventaire de Bourgogne en 2011, Patrimoine hospitalier en Bourgogne ${ }^{1}$, et celle, nationale, de l'Inventaire général du patrimoine culturel, l'année suivante, L'Hôpital en France, histoire et architecture ${ }^{2}$. En octobre 2013, année de commémoration du tricentenaire de la naissance de l'architecte Jacques-Germain Soufflot, à qui l'on doit notamment l'hôtelDieu de Lyon et l'hospice de la Charité à Mâcon, les associations GHAMU (groupe Histoire, Architecture, Mentalités urbaines) ${ }^{3}$ et MOHICAN (association nationale des chargés de protection au sein des conservations régionales des Monuments historiques) ont uni leurs forces afin d'organiser des journées d'étude intitulées «Patrimoine et santé : de Soufflot à nos jours »4. Parallèlement à l'actualité de la recherche, l'actualité patrimoniale est fortement marquée par des problématiques de protection ou de reconversion d'établissements hospitaliers souvent anciens, (hôtels-Dieu de Paris, Lyon et Dijon), parfois très récents (CHU de Caen), et par un débat plus large sur le devenir de bâtiments liés à la santé (sanatoriums d'Aincourt dans le Val-d'Oise, ou de Bergesserin en Saône-et-Loire), mais aussi sur les difficultés de la présentation au public des collections d'objets et d'instruments médicaux (fermeture en 2012 du musée de l'Assistance publique-Hôpitaux de Paris). 
Figure 1

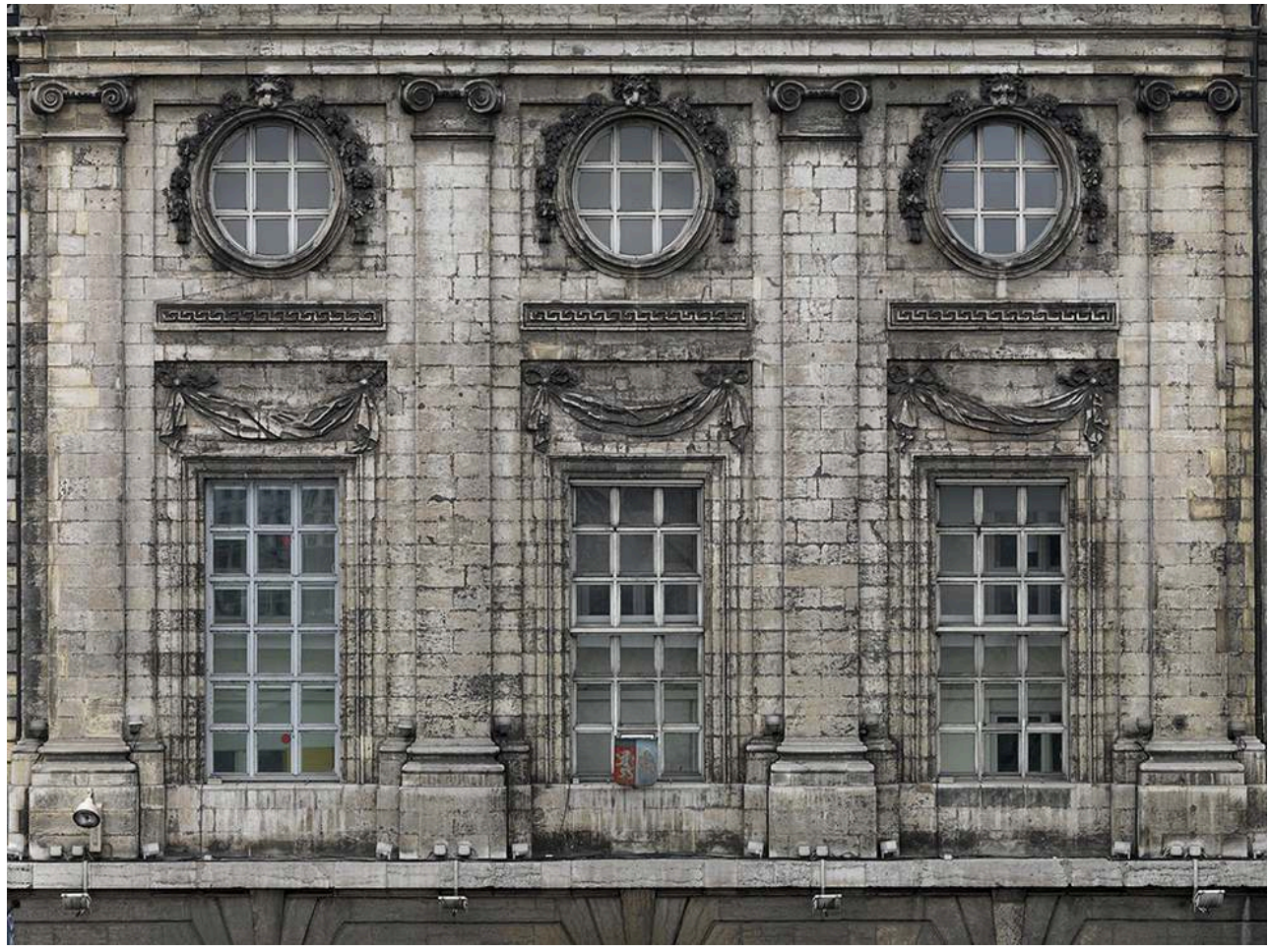

DÉTAIL de LA FAÇADE de L'HôTEL-DIEU de LYON (RHôNE).

Phot. Dessert, Éric. (C) Région Rhône-Alpes, Inventaire général du patrimoine culturel, 2010

2 C'est pourquoi le comité de lecture a retenu la proposition d'un numéro thématique construit à partir des communications faites au cours de ces trois journées d'étude tout en élargissant le périmètre du sujet grâce à un appel à contributions. Le colloque de 2013 avait pour ambition de réunir les points de vue d'universitaires, de chercheurs et de professionnels $\mathrm{du}$ patrimoine. Oscillant entre histoires architecturales et expériences de terrain, il s'articulait autour de trois thèmes: les évolutions de l'architecture consacrée à la santé du XVIII ${ }^{\mathrm{e}}$ siècle à nos jours, la politique de protection au titre des monuments historiques et la reconversion, enfin, les objets mobiliers et décors. Comme pour les journées d'étude, l'objectif de l'appel à contributions d'In Situ était de rassembler et de croiser les approches des chercheurs académiques (doctorants en histoire ou en histoire de l'art, enseignants-chercheurs) avec celles des professionnels du patrimoine (chercheurs de l'Inventaire général, responsables des Monuments historiques, archivistes, conservateurs de musées ou architectes), dans une perspective transdisciplinaire. Il s'agissait de prendre en compte tous les biens culturels potentiellement concernés, l'architecture bien entendu, mais également les objets mobiliers (œuvres d'art au sens traditionnel du terme ou collections scientifiques et techniques), les archives ou encore les pratiques culturelles immatérielles. En outre, il semblait essentiel d'étendre cette approche à l'ensemble des patrimoines liés à la santé, en ne se limitant pas à l'hôpital stricto sensu mais en explorant les pistes du patrimoine sanitaire et social. Cet appel a connu un franc succès - autre signe de l'importance et de l'actualité du sujet dans les mondes universitaires et patrimoniaux et le présent numéro compte quarante articles. 
Figure 2

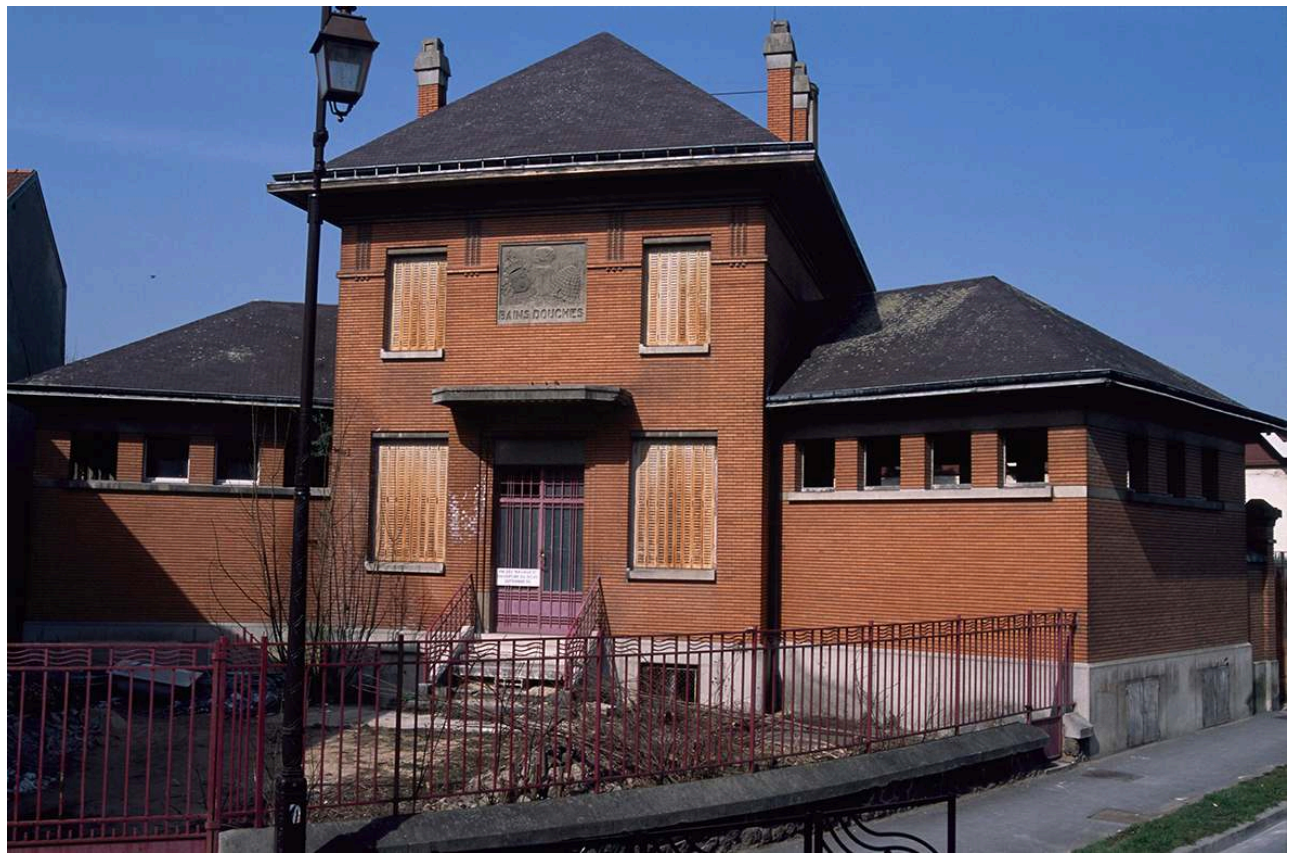

ÉTABLISSEMENT DE BAINS-DOUCHES, ÉPERNAY.

Phot. Delance, P. (c) Région Alsace Champagne-Ardenne Lorraine, Inventaire général du patrimoine culturel, 2004.

3 Il est organisé en deux grandes parties. La première s'attache à définir une typologie des différents patrimoines liés à la santé. La seconde s'intéresse plus particulièrement aux enjeux de leur conservation et de leur valorisation. Volontairement didactique, cette organisation thématique n'est pas totalement étanche et l'on pourra trouver des considérations typologiques dans la partie consacrée à la conservation et inversement.

\section{Essais de définition}

Cette première partie appréhende les patrimoines de la santé dans la diversité de leurs modèles, de leurs fonctions ou de leurs sources. 
Figure 3

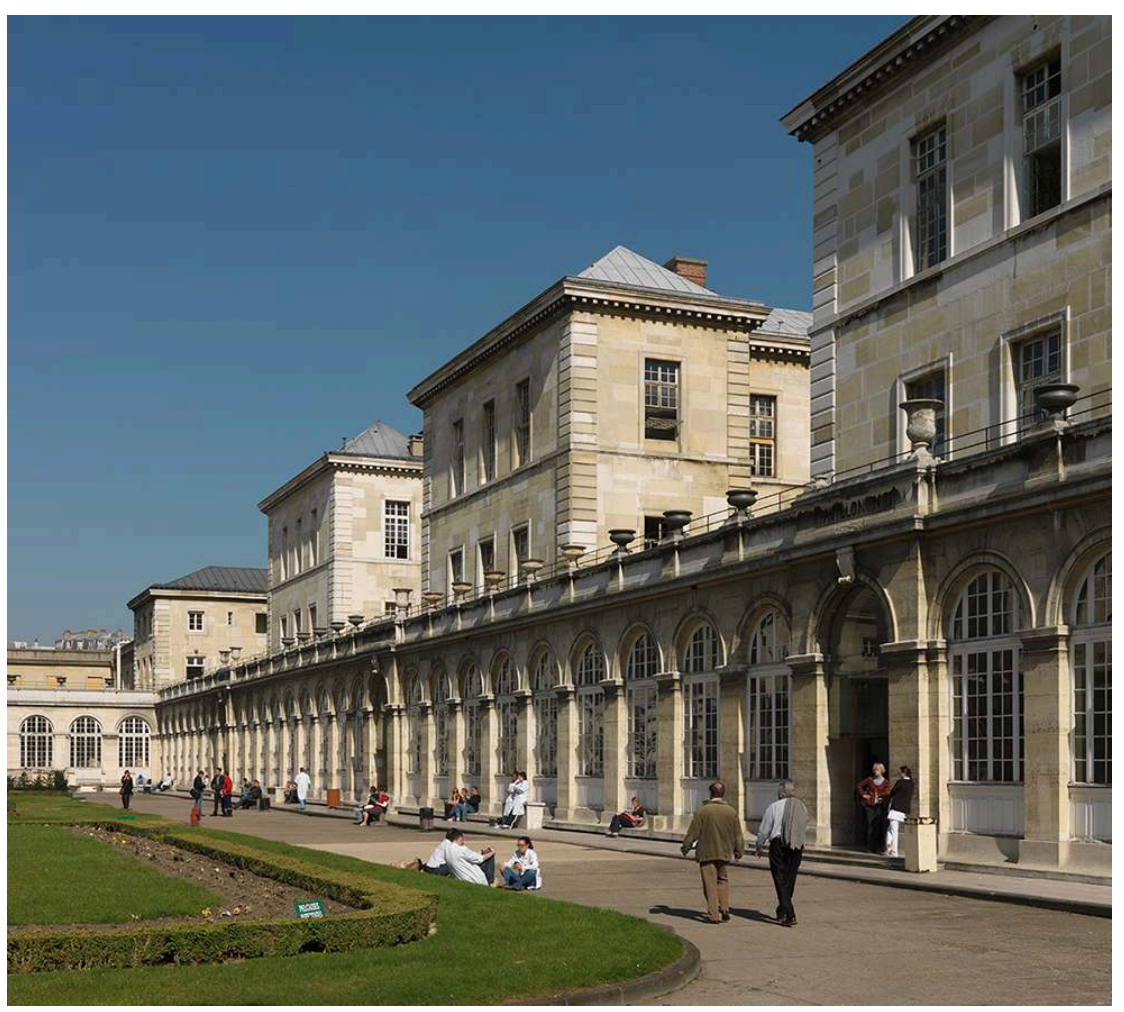

L'HÔPITAL LARIBOISIÈRE, PARIS.

Phot. Kruszyk, L. @ Région Île-de-France, Inventaire général du patrimoine culturel, 2012.

Parmi les articles, ceux ayant trait aux hôpitaux et aux hôtels-Dieu occupent une place importante et présentent des exemples qui vont de l'Ancien Régime au XX $\mathrm{XX}^{\mathrm{e}}$ siècle. Des réflexions théoriques des Lumières aux conceptions d'hôpitaux " modernes ", on peut mesurer à quel point ce type est complexe à appréhender pour l'historien. En effet, en dehors des grands gestes architecturaux que peuvent constituer l'hospice de la Charité de Mâcon (1752-1762) par Jacques-Germain Soufflot ou l'hôpital-mémorial de Saint-Lô (1949-1956) par Paul Nelson, la plupart des ensembles hospitaliers sont formés d'éléments composites, juxtaposant des bâtiments élevés à des époques différentes et s'adaptant aux contraintes du terrain, des commanditaires et des évolutions de la science et de la médecine, comme le démontre l'article sur les équipements rennais. Sans oublier les édifices transformés provisoirement en établissements de soins tels les hôpitaux militaires de la Grande Guerre évoqués à travers l'exemple d'Aix-les-Bains (Savoie). 
Figure 4

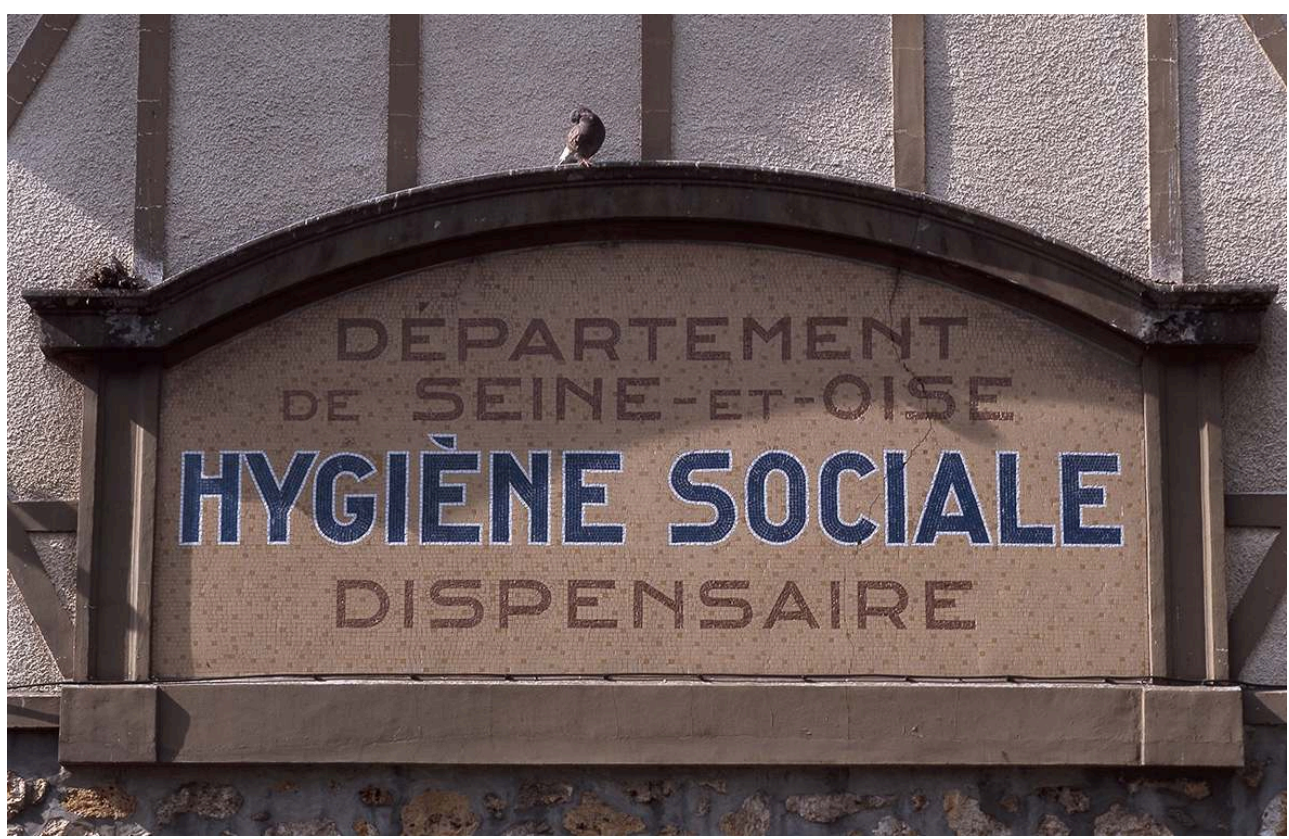

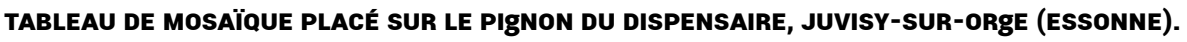

Phot. Ayrault, P. () Région Île-de-France, Inventaire général du patrimoine culturel, 2005.

6 L'hôpital, aussi symbolique soit-il, n'étant que l'élément le plus visible, nous avons souhaité élargir cette approche thématique à l'ensemble des réalisations relevant de la catégorie de «l'architecture hospitalière d'assistance ou de protection sociale » afin d'en mieux cerner les contours ${ }^{5}$. Cette catégorie comprend aussi bien les sanatoriums certes bien étudiés par ailleurs dans les synthèses de Philippe Grandvoinnet ou de JeanBernard Cremnitzer ${ }^{6}$ - mais également les hospices marins, les dispensaires antituberculeux ou encore les bâtiments de transit pour émigrants, très peu documentés jusqu'à la thèse d'Anne Bosser. Quant aux établissements de bains ou aux bâtiments thermaux, ils sont davantage connus pour leurs caractéristiques liées à la villégiature ou aux loisirs que pour leurs qualités dans le domaine de l'hygiène ou leurs équipements curatifs. Deux textes les présentent sous un jour nouveau. Monumentaux ou plus modestes, tous ces édifices ont marqué les consciences et les territoires européens au cours des $\mathrm{XIX}^{\mathrm{e}}$ et $\mathrm{xx}^{\mathrm{e}}$ siècles: devenus obsolètes, ils sont détruits et disparaissent ainsi lentement $d u$ paysage. Lorsqu'ils sont transformés pour de nouveaux usages, ils perdent trop souvent le sens de leur vocation initiale aux yeux du monde. 


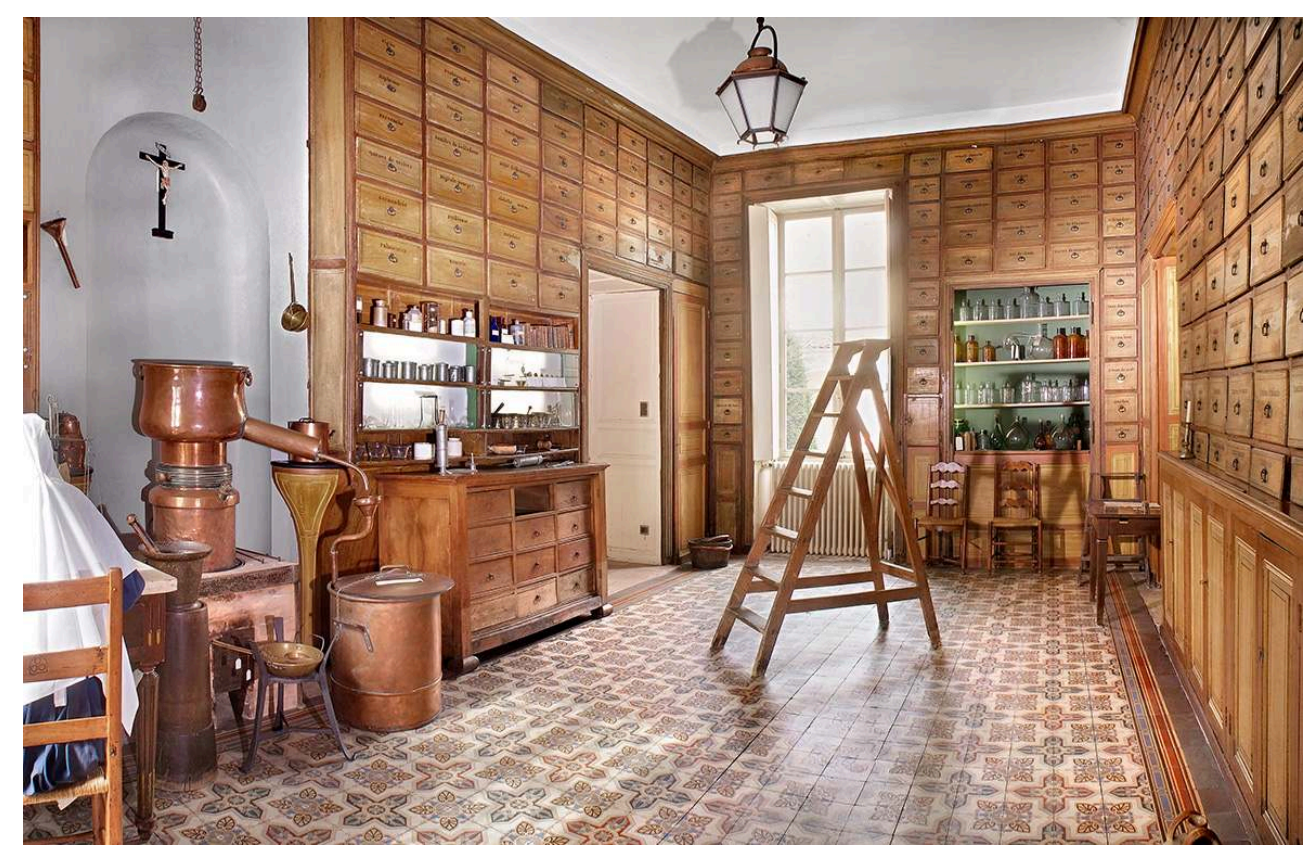

PHARMACIE de L'HÔPITAL SAINT-LAURENT (ACTUEL CENTRE HOSPITALIER WILLIAM-MOREY), CHALONSUR-SAÔNE (SAÔNE-ET-LOIRE).

Phot. Kuntz, T. (c) Région Bourgogne, Inventaire général du patrimoine culturel, 2008.

Une troisième sous-partie évoque les objets, dans toute leur diversité, ceux ayant directement trait aux soins autant que ceux qui incarnent l'institution hospitalière. La synthèse régionale bourguignonne citée plus haut avait bien mis en exergue la richesse et la diversité de ces ensembles, quelle que soit leur époque. Songeons aux splendides apothicaireries des hôpitaux de Chalon-sur-Saône, de Mâcon ou de Tournus, véritables écrins d'objets à l'origine utilitaires, considérés aujourd'hui comme des objets d'art. Un article présente l'atelier de Toine Coivet, fournisseur de mortiers en bronze pour les apothicaireries du Lyonnais au XVII siècle; un second aborde la figure emblématique des donateurs à travers les collections de portraits conservés dans quelques établissements hospitaliers bourguignons. Mais la définition des patrimoines mobiliers de la santé présente actuellement des contours encore mouvants tant le patrimoine scientifique reste difficile à cerner. Ainsi, un texte nous fait découvrir les machines orthopédiques que le Dr François Humbert conçut durant la première moitié du XIX siècle. Auraient pu être évoquées ici bien d'autres collections médicales ou chirurgicales, telles celle du récent conservatoire du patrimoine hospitalier de Rennes (CPHR) fondé en $2011^{7}$ ou le musée d'anesthésie-réanimation et des techniques médicochirurgicales installé dans l'ancien hôtel-Dieu de Besançon, dans le bloc opératoire conçu par André Walter en 1935 et protégé au titre des monuments historiques en $2012^{8}$. D'autres encore sont abordées dans la seconde partie du numéro. 


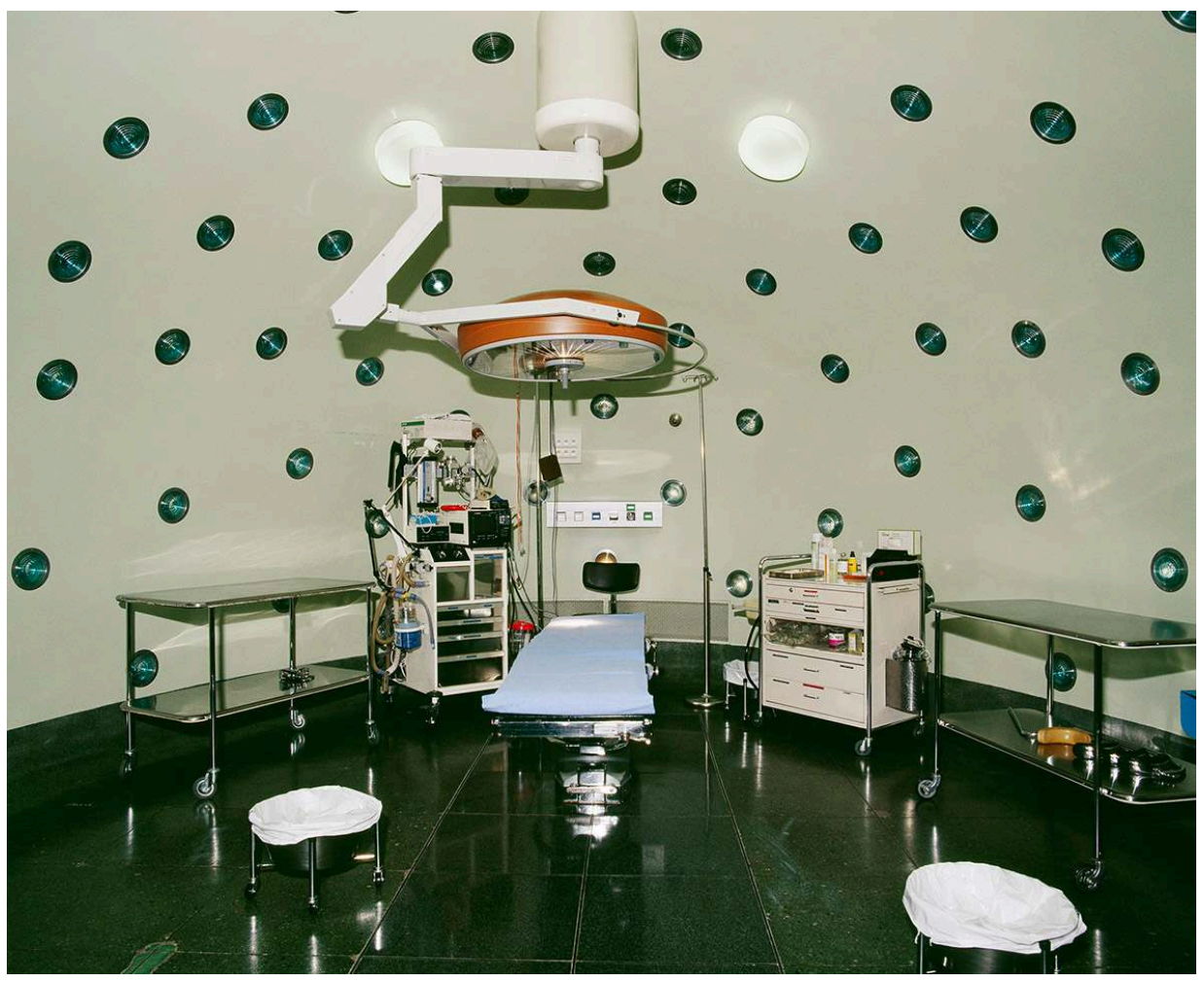

SALLE d'OPÉRATION, HÔPITAL-MÉMORIAL FRANCE - ÉTATS-UNIS CONSTRUIT PAR PAUL NELSON, SAINT-LÔ (MANCHE).

Phot. Corbière, P. @ Région Normandie, Inventaire général du patrimoine culturel, 1994.

Enfin, la question des sources imprimées et des archives est également étudiée. Documents essentiels à la bonne compréhension de l'architecture des bâtiments, elles apportent des informations primordiales pour appréhender le fonctionnement de tous ces équipements et les nécessaires évolutions que médecins et architectes ont pu imaginer afin de moderniser ces « machines à guérir » qu'étaient devenus les hôpitaux dès la seconde moitié du XIX ${ }^{e}$ siècle. Pierre-Louis Laget, éminent spécialiste français de l'histoire architecturale hospitalière, expose dans un texte introductif les spécificités des sources imprimées de ce champ d'étude et alerte sur les risques que ces particularismes font courir à la recherche. L'analyse de quelques fonds des archives départementales du Val-d'Oise - de l'ancienne DDASS et de petites communes - apporte un éclairage inédit sur les politiques nationales de santé depuis le $\mathrm{XIX}^{\mathrm{e}}$ siècle déclinées à une échelle locale. Enfin, la numérisation des recueils des travaux du Comité consultatif d'hygiène publique évoquée dans un dernier article met à disposition de la communauté des chercheurs des sources clés de cette politique publique française.

\section{Enjeux de conservation}

9 La seconde partie de ce numéro aborde les enjeux liés à l'étude, la protection, la réutilisation et la valorisation de ces biens culturels.

Leur reconnaissance patrimoniale n'a pas toujours constitué une évidence et reste un objectif à atteindre pour certaines catégories d'entre eux. En effet, les ensembles architecturaux ont été conçus pour répondre à des besoins sanitaires et accueillent 
encore des activités de soin. D'autres ont changé d'affectation, contraints par l'évolution des normes médicales ou les réorganisations successives de la carte hospitalière. Quant aux objets, si les œuvres d'artistes sont identifiées depuis longtemps, ceux du quotidien médical, anciens comme récents, peinent à susciter un intérêt quelconque auprès d'un public autre que scientifique. Toutes ces raisons, auxquelles s'ajoutent les difficultés financières récurrentes des établissements, expliquent la lenteur de leur patrimonialisation. Les efforts conjugués des chercheurs et de nombreux services patrimoniaux contribuent cependant, grâce aux études qu'ils conduisent, à faire émerger leur valeur patrimoniale: qu'il s'agisse de la collecte d'archives orales auprès des sœurs hospitalières de l'hôtel-Dieu de Beaune ou de l'inventaire du patrimoine immatériel hospitalier en passant par l'étude de collections pédagogiques tombées en désuétude, telles celles de cires anatomiques, le patrimoine de la santé voit ses contours s'agrandir et se préciser. C'est ainsi qu'il peut être le mieux défendu et protégé.

Figure 7

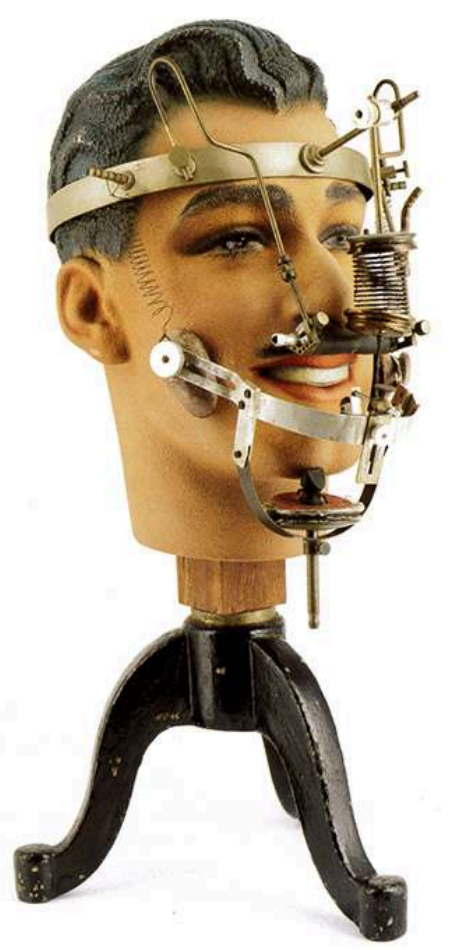

CASQUE UTILISÉ POUR MENER DES RECHERCHES EN PHONÉTIQUE CONÇU PAR LE PHONIATRE HENDRIK ZWAARDEMAKER (1857-1930). CET APPAREIL PERMETTAIT D'ENREgISTRER LE MOUVEMENT DE LA LÈVRE SUPÉRIEURE ET DE LA MÂCHOIRE INFÉRIEURE DURANT UN DISCOURS.

(C) Collection de l'Université d'Amsterdam.

11 L'inscription ou le classement au titre des monuments historiques semble la solution idéale pour sauvegarder un tel héritage. Dominique Perrin et Frantz Schoenstein résument l'histoire et l'évolution de la protection des hôpitaux et des motifs qui y présidèrent. Les intérêts historiques et esthétiques ont toujours été importants mais le critère mémoriel peut se révéler tout aussi décisif. C'est le cas pour la maternité suisse d'Elne (Pyrénées-Orientales), classée entièrement en 2013, qui accueillit de 1939 à 1944 des parturientes réfugiées espagnoles, regroupées dans les camps d'internement du sud 
de la France. Un constat s'impose cependant: sans projet de reconversion viable, notamment pour les édifices désaffectés, ou de moyens financiers substantiels, comme pour tout autre type de patrimoine, la protection au titre des monuments historiques ne suffit pas à garantir une bonne conservation. Elle peut par ailleurs constituer une entrave à la modernisation de bâtiments toujours en activité : ces éléments de contexte avaient été largement évoqués par les chargés de la protection en fonction dans les DRAC lors des journées d'étude de 2013.

Figure 8

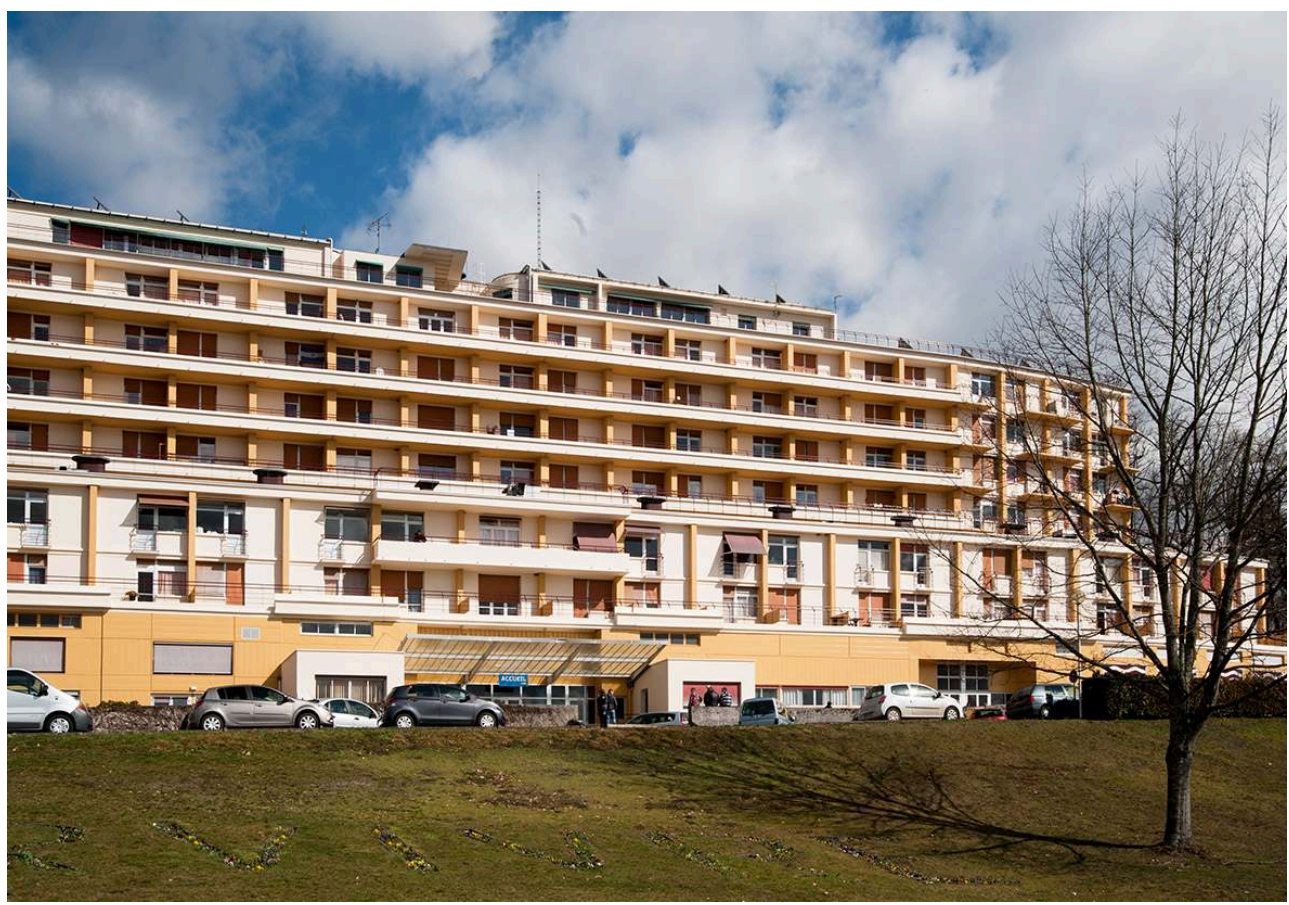

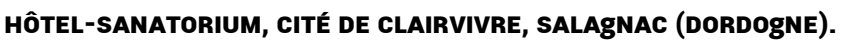

Phot. Dubau, M. (c) Région Aquitaine, Inventaire général du patrimoine culturel, 2012.

12 Effectivement, étape délicate pour tout édifice historique, la question de la reconversion est abordée dans plusieurs articles. Qu'il s'agisse d'anciens hôpitaux ou de sanatoriums, les projets semblent plus aisés à mettre en œuvre dans un espace urbain que dans un territoire rural ou une zone excentrée. De nombreux exemples "réussis » témoignent d'une concertation intelligente des acteurs publics et privés : c'est le cas des hôpitaux de Cholet (Maine-et-Loire) ou de Montpellier (Hérault). D'autres achoppent par manque de moyens financiers et humains, ou tout simplement parce que le site ou le bâtiment ne s'y prêtent que très difficilement: c'est ce que Philippe Grandvoinnet tente de démontrer à propos de la reconversion des sanatoriums de cure en France. L'agence de programmistes $A$ et cetera explique quant à elle les enjeux urbains et politiques d'une méthode de conduite de projet de reconversion impliquant les citoyens. Aujourd'hui, la concertation, et au-delà, la démocratie participative, l'innovation urbaine et culturelle sont à ses yeux les conditions nécessaires au succès de la transformation des plus vastes emprises hospitalières des cœurs de ville. 


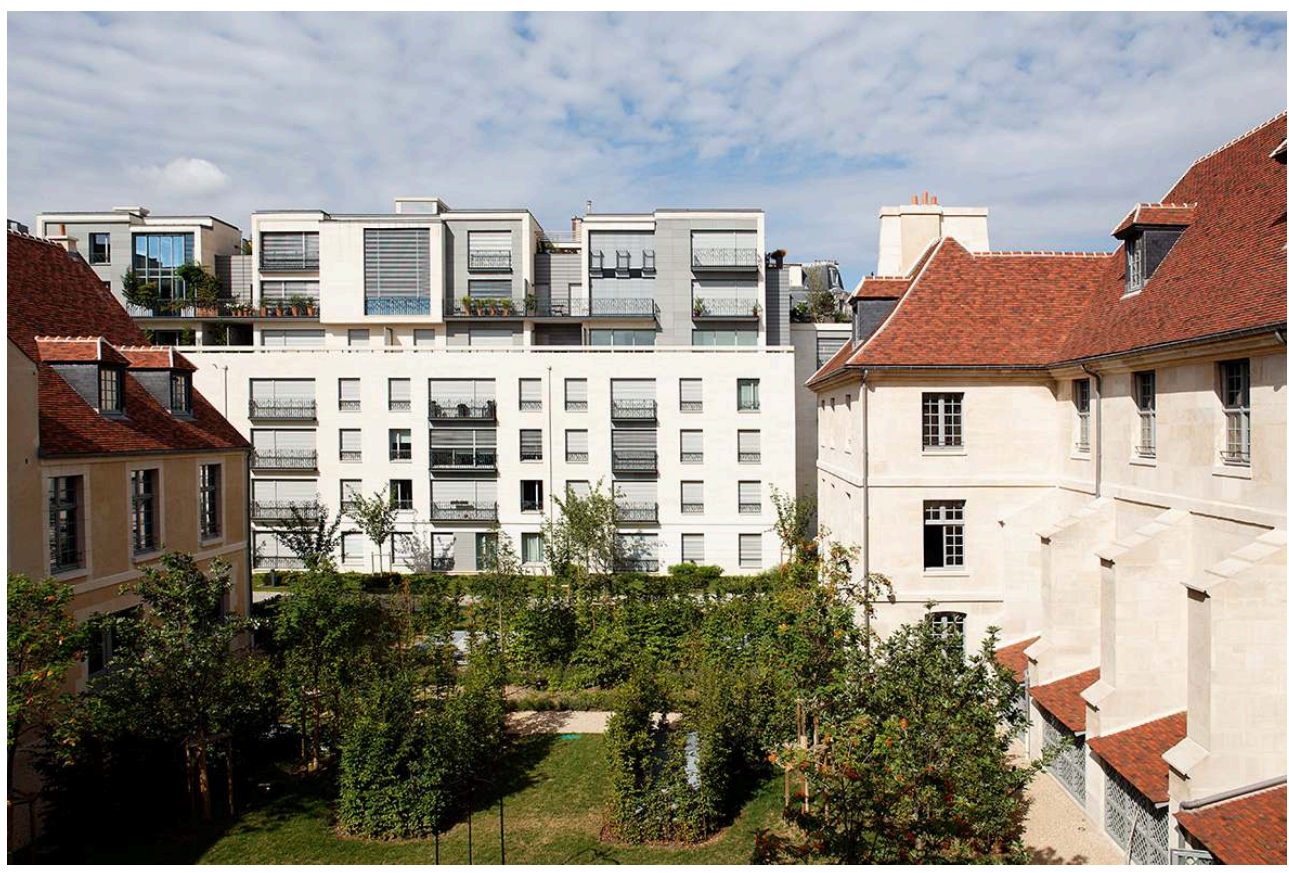

ANCIEN HÔPITAL LAENNEC, PARIS. AU FOND, UN IMMEUBLE DE LOgEMENTS CONÇU PAR L'AgENCE D'ARCHITECTURE VALODE ET PISTRE.

(C) Valode \& Pistre architectes / Phot. Daniel Moulinet.

Les opérations de valorisation font l'objet d'un ultime développement dans ce numéro. Elles sont incontournables pour sensibiliser le public à ces patrimoines souvent peu accessibles, au propre comme au figuré. Qu'il s'agisse de la création de musées au sein d'hôpitaux, comme celui de l'hôtel-Dieu de Château-Thierry (Aisne), ou de médiations dans des établissements encore en activité, comme à l'asile de Pau, elles rencontrent un certain succès ; comme pour les reconversions, elles nécessitent cependant des moyens qui ne sont pas toujours disponibles en période de crise et une entente entre les divers acteurs que sont l'établissement propriétaire, les collectivités locales, les associations de défense du patrimoine et les professionnels de la Culture.

Figure 10

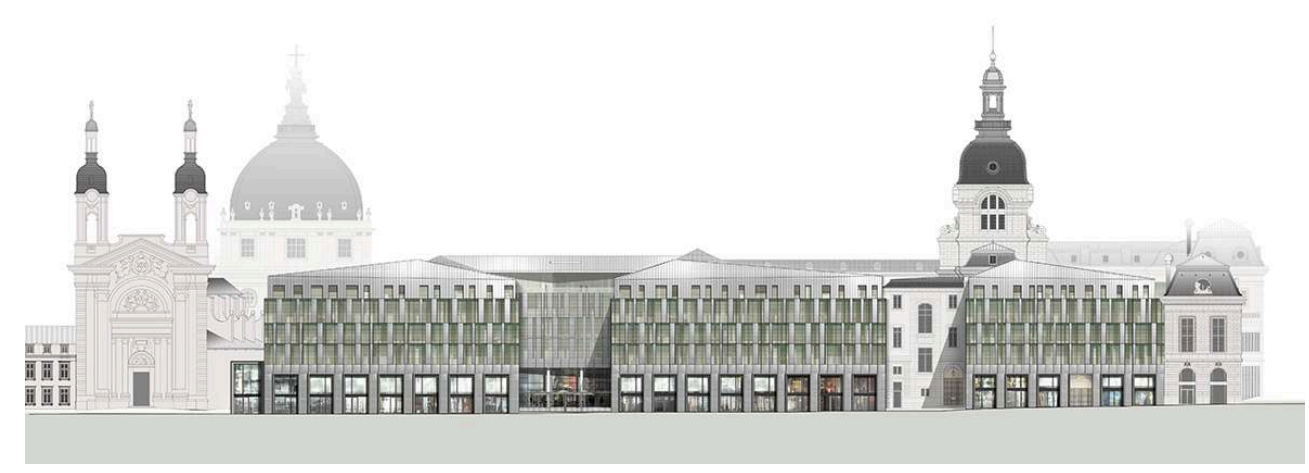

RECONVERSION DE L'HôTEL-DIEU DE LYON (RHÔNE), ÉLÉVATION DE LA NOUVELLE FAÇADE SUR LA RUE BELLECORDIÈRE PAR ALBERT CONSTANTIN ET AIA ASSOCIÉS

(C) AIA associés. 
Ce $31^{\mathrm{e}}$ numéro d'In Situ. Revue des patrimoines n'épuise pas son sujet. Les opérations urbaines - de la reconversion à la destruction, renommée à cette occasion « déconstruction " pour paraître, peut-être, moins violente - s'enchaînent, au Vésinet (Yvelines), à Lyon (Rhône), à Saint-Germain-en-Laye (Yvelines), à Lorient (Morbihan), à Saint-Dizier (Haute-Marne), à Toulon (Var) ou encore à Metz (Moselle)... Et le phénomène va se poursuivre. Après que l'instauration d'une carte sanitaire a conduit à la fermeture de plus de 80000 lits entre 1992 et 2003, la nouvelle loi « de modernisation de notre système de santé" promulguée le 26 janvier 2016 oblige dorénavant les 850 hôpitaux publics à rejoindre l'un des 130 groupements hospitaliers de territoire (GHT) afin d'y développer coopérations, mutualisations et in fine, économies d'échelle9. Par ailleurs, la "révolution ambulatoire " en cours entraînera mécaniquement une surcapacité de 35000 lits dans les cinq prochaines années ${ }^{10}$. Ces bouleversements sont le prélude à des restructurations futures plus profondes. Cependant, le nombre de nouvelles protections au titre des monuments historiques reste modeste (à peine une douzaine depuis 2010). Même l'attribution du label «Patrimoine $\mathrm{du} \mathrm{xx}^{\mathrm{e}}$ siècle " ne semble pas aller de soi : en quinze années d'existence, cette distinction - pourtant dénuée de toute contrainte juridique - n'a été attribuée qu'à six hôpitaux et une dizaine de sanatoriums ${ }^{11}$. Il est nécessaire que la diffusion des connaissances et les actions de valorisation se poursuivent. C'est la raison pour laquelle une réédition mise à jour de l'ouvrage L'Hôpital en France, histoire et architecture, déjà épuisé, a été réalisée à la fin de l'année 2016. Les recherches doivent également se poursuivre afin d'étayer les futures protections et les opérations de requalification. Par ailleurs, l'extension esquissée dans ce numéro du champ des patrimoines de la santé à certains aspects du thermalisme ou de pratiques de loisirs (colonies de vacances) ouvre de nouvelles pistes de travail qui ne manqueront pas de donner lieu à un second numéro thématique.

\section{NOTES}

1. - Bourgogne, service Patrimoine et Inventaire; HUGONNET-BERGER, Claudine, REVEILLON, Élisabeth, FROMAGET, Brigitte (réd.). Patrimoine hospitalier en Bourgogne. Paris : Somogy, 2011.

2. - France, Inventaire général du patrimoine culturel ; LAGET, Pierre-Louis, LAROCHE, Claude, DUHAU, Isabelle (dir.). L'Hôpital en France. Histoire et architecture. Lyon : Lieux-Dits, coll. « Cahiers du Patrimoine ; $99 », 2012$.

3. - Le GHAMU est une association de chercheurs, professionnels et amateurs du patrimoine qui s'intéressent particulièrement à l'histoire de l'architecture, de la création artistique, de la vie urbaine et des jardins de la période moderne. http://www.ghamu.org/.

4. - Le tricentenaire de la naissance de Jacques-Germain Soufflot (1713-1780), inscrit parmi les célébrations nationales de 2013, a été l'occasion pour le GHAMU d'organiser un colloque à deux facettes. La première, les journées d'étude en Bourgogne, en partenariat avec l'association MOHICAN, intitulées «Patrimoine et santé : de Soufflot à nos jours», était soutenue par le ministère de la Culture et de la Communication à travers la mission de l'Inventaire général du patrimoine culturel et la direction régionale des Affaires culturelles de Bourgogne. La seconde, une journée d'étude parisienne, «Jacques-Germain Soufflot ou l'architecture régénérée

In Situ, 31 | 2017 
(1713-1780) », a vu ses actes publiés en 2015 aux éditions Picard (sous la direction de Claire Ollagnier et Daniel Rabreau).

5. - Au sens du vocabulaire de l'Inventaire général du patrimoine culturel. Voir le thésaurus de la désignation des œuvres architecturales et des espaces aménagés: http://data.culture.fr/ thesaurus/page/ark:/67717/T96-269.

6. - CREMNITZER, Jean-Bernard. Architecture et santé. Le temps du sanatorium en France et en Europe. Paris : Picard, 2005 ; GRANDVOINNET, Philippe. Architecture thérapeutique. Histoire des sanatoriums en France (1900-1945). Genève : MétisPresses, 2014.

7. - Voir le site : http://www.cphr.fr/ [consulté le 21/02/2017].

8. - Voir le site : http://www.chu-besancon.fr/museum/ [consulté le 21/02/2017].

9. - Le Monde, 12 juillet 2016.

10. - Le Monde, 2 mai 2016.

11. - Ces chiffres ne comptabilisent pas les édifices $d u x^{e}$ siècle protégés au titre des monuments historiques qui ont également, de facto, reçu le label (soit une quinzaine d'hôpitaux ou de sanatoriums). Voir le site: http://www.culturecommunication.gouv.fr/Aides-demarches/ Protections-labels-et-appellations/Label-Patrimoine-du-XXe-siecle.

\section{AUTEURS}

\section{ISABELLE DUHAU}

Chargée de mission, Mission Inventaire général du patrimoine culturel, Service du Patrimoine, Direction générale des Patrimoines isabelle.duhau@culture.gouv.fr

\section{CÉCILE LESTIENNE}

Attachée de conservation du patrimoine, Responsable de l'Inventaire du patrimoine, archives départementales du Val-d'Oise cecile.lestienne@valdoise.fr 